\title{
The Dynamic Gravitation of Photons from the Perspective of Maxwell's Wave Equations
}

\author{
Guido Zbiral \\ Private Retired Scientist, Klosterneuburg, Austria \\ Email: guido@zbiral.at
}

Received 14 May 2014; revised 8 June 2014; accepted 2 July 2014

Copyright (C) 2014 by author and Scientific Research Publishing Inc.

This work is licensed under the Creative Commons Attribution International License (CC BY).

http://creativecommons.org/licenses/by/4.0/

(c) (i) Open Access

\begin{abstract}
Although the gravitational constant $(G)$ does not explicitly occur in the Maxwell Wave Equations, this paper will show that $G$ is indeed implicitly contained in them. The logical consequence hereby is that electromagnetic radiation is associated with dynamic gravitation and not-as assumed in Einstein's Special Theory of Relativity-with "static" gravitation, dynamic gravitation being at the time unknown. According to the Maxwell Wave Equations, gravitation experiences the same dynamic (speed of light $c$ ) as electromagnetic radiation and must therefore also be of a quantum nature. There must exist an equal number of gravitational quanta as there are photons. Since photons do not possess a baryonic rest mass but only a relativistic mass, this mass must be nonbaryonic in nature-precisely as their dynamic gravitation.
\end{abstract}

\section{Keywords}

Photon, Dynamic Gravitation, Gravitational Quanta, Maxwell's Wave Equations

\section{Introduction}

For more detailed information on the nature of dynamic gravitation of photons, please refer to the paper by Guido Zbiral: The "Dynamic Gravitation of Photons: A Hitherto Unknown Physical Quantity". New Aspects on the Physics of Photons in Journal of Modern Physics, 5, 198-204. http://dx.doi.org/10.4236/jmp.2014.55030 (March 2014).

The following short paper is intended as a supplement to the paper cited above. 


\section{The Dynamic Gravitation of Photons from the Perspective of Maxwell's Wave Equations}

The Maxwell's Wave Equations for the $x$-axis are: [1] [2]

$$
\begin{gathered}
\delta^{2} E / \delta x^{2}=\mu_{0} \varepsilon_{0} * \delta^{2} E / \delta t^{2} \text { or } 1 / c^{2} * \delta^{2} E / \delta t^{2} \\
\delta^{2} B / \delta x^{2}=\mu_{0} \varepsilon_{0} * \delta^{2} B / \delta t^{2} \text { or } 1 / c^{2} * \delta^{2} B / \delta t^{2}
\end{gathered}
$$

The vectors for the electrical component $E$ and the magnetic component $B$ of the electromagnetic wave are perpendicular to the direction of propagation (the $x$-axis) and are thus transversal waves; furthermore, the $E$ field and the $B$-field are themselves mutually perpendicular (along the $y$ - and $z$-axes, respectively); both fields are completely symmetrical and also - in regard to their energy — completely equivalent. The dynamic E-field and the dynamic B-field are inseparably linked to each other in the electromagnetic wave and are mutually dependent.

Since the property $c^{2}\left[\mathrm{~m}^{2} \cdot \mathrm{s}^{-2}\right]$ is included in the dimensions of the Gravitational Constant $G=6.67 \times 10^{-11}$ $\left[\mathrm{m}^{3} \cdot \mathrm{kg}^{-1} \cdot \mathrm{s}^{-2}\right]$, and according to the so-called Maxwell's 5th Equation:

$$
\varepsilon_{0} * \mu_{0}=1 / c^{2}
$$

the following relationship then applies:

$$
G / c^{2}=G * \varepsilon_{0} * \mu_{0}=(\text { Constant })=7.42 \times 10^{-28}\left[\mathrm{~m} \cdot \mathrm{kg}^{-1}\right] \text { respectively } \varepsilon_{0} * \mu_{0}=C / G
$$

When the relationship (4) is inserted into the two Maxwell Wave Equations (1), (2), they then become:

$$
\begin{aligned}
& \delta^{2} E / \delta x^{2}=C / G * \delta^{2} E / \delta t^{2} \\
& \delta^{2} B / \delta x^{2}=C / G * \delta^{2} B / \delta t^{2}
\end{aligned}
$$

Both of these converted wave equations now contain the Gravitational Constant $G$ and can be interpreted as follows:

Since the two dynamic vector fields $E$ and $B$ transport electrical and magnetic energy along their respective axes with them, each of these fields are associated with $G$, i.e., both components of the electromagnetic wave are subject to gravitation.

Gravitation (denoted by the constant physical value $G$ ) is not able to create a gravitational wave (i.e. radiation) of its own accord. However, as $G$ is inseparably associated with both the dynamic vector fields $E$ and $B^{1}$ gravitation is - so to speak- “carried along” by the two vector fields to each other. Due to its coupling with $E$ and $B$, gravitation must of necessity assume all the dynamic properties possessed by $E$ and $B^{2}$. Gravitation is thus propagated at the speed of light along the $x$-axis with the same frequency $v$ as the electromagnetic wave. For this reason, gravitation must possess a wave property-i.e. radiation of a quantum nature.

Gravitational waves are-as is the case with electromagnetic waves-transverse waves and resonate synchronously in the plane of their respective field components $E$ and $B$. Since $G$ is expressed completely symmetrically in both of Maxwell's Wave Equations (5), (6), the gravitational waves also obey Maxwellian theory in this regard. It therefore follows that the equation for the energy of the electromagnetic radiation (of the photons)

$$
E=h \cdot v
$$

also applies to the gravitational radiation (of the gravitational quanta) associated with the photons. Gravitational waves are therefore completely equivalent to the $E$ - und $B$-fields of electromagnetic waves, albeit acting in an opposite manner. At the constant speed of light, a stable state of equilibrium exists within each photon between the expansive force of electrodynamics and the equal but opposing (braking) force of its dynamic gravitation. Therefore at the constant speed of light, the resulting total energy of every photon is always zero! This is an absolute necessity for the constancy of the speed of light.

This derivation represents a confirmation that photons as dynamic electromagnetic quanta are inseparably linked to dynamic gravitation (in the form of gravitational quanta).

\footnotetext{
${ }^{1} E$ and $B$ are energy fields; each form of energy is inseparably associated with gravitation.

${ }^{2}$ The properties of the dynamic vector fields $E$ and $B$ are-as it were-transferred to the gravitation.
} 


\section{Conclusion}

The existence of dynamic gravitation, proposed in the aforementioned paper, is verified as a result of the theoretical derivation from the Maxwell Wave Equations.

\section{Acknowledgements}

My warmest thanks go to my translator Kris Szwaja (M.A. Physics, Oxon), both for translating my manuscript from German into English and his valuable suggestions on the text itself.

\section{References}

[1] Leisen, J. Research Seminar on “Die Maxwell-Gleichungen Verstehen”, University of Mainz.

[2] (2004) Die Maxwell-Gleichungen und ihre Bedeutung für die SRT. http://www.mahag.com/srt/maxwell.php

[3] Wikipedia (Google) (2014) Article on the Higgs Mechanism.

\section{Appendix}

In the review comments of this paper, the opinion was expressed that the dynamic gravitation of photons possesses an internal connection with the source of the (relativistic) mass of these fundamental particles, which could possibly be interpreted by the Higgs Mechanism (or Higgs field).

In this regard the following should be noted: All elementary particles with baryonic mass obtain their mass by means of interaction with the Higgs Field, whereby a "non-relativistic environment" is supposed. The Higgs Field is massive but not directly measurable; the associated Higgs boson has a mass of approx. $125 \mathrm{GeV}$ and acts purely "longitudinally".

In contrast, electromagnetism is not of massive nature and the Higgs Mechanism does not couple with it. For this reason the gauge boson relevant for this theory, the photon, possesses no rest mass, it does, however, possess a relativistic mass and displays a purely "transversal" behaviour. While the dynamic gravitation of photons is associated with its relativistic mass and exists only within a relativistic environment, for the reasons described above it is not possible to establish a relationship of the relativistic mass with the Higgs Mechanism. This means that from the present-day perspective, the relativistic mass of photons cannot be explained by means of the Higgs Mechanism - there must be a different cause at work.

It has not yet been definitively proven whether the new particle discovered in July 2012 is indeed the Higgsboson predicted in the Standard Model [3]. 
Scientific Research Publishing (SCIRP) is one of the largest Open Access journal publishers. It is currently publishing more than 200 open access, online, peer-reviewed journals covering a wide range of academic disciplines. SCIRP serves the worldwide academic communities and contributes to the progress and application of science with its publication.

Other selected journals from SCIRP are listed as below. Submit your manuscript to us via either submit@scirp.org or Online Submission Portal.
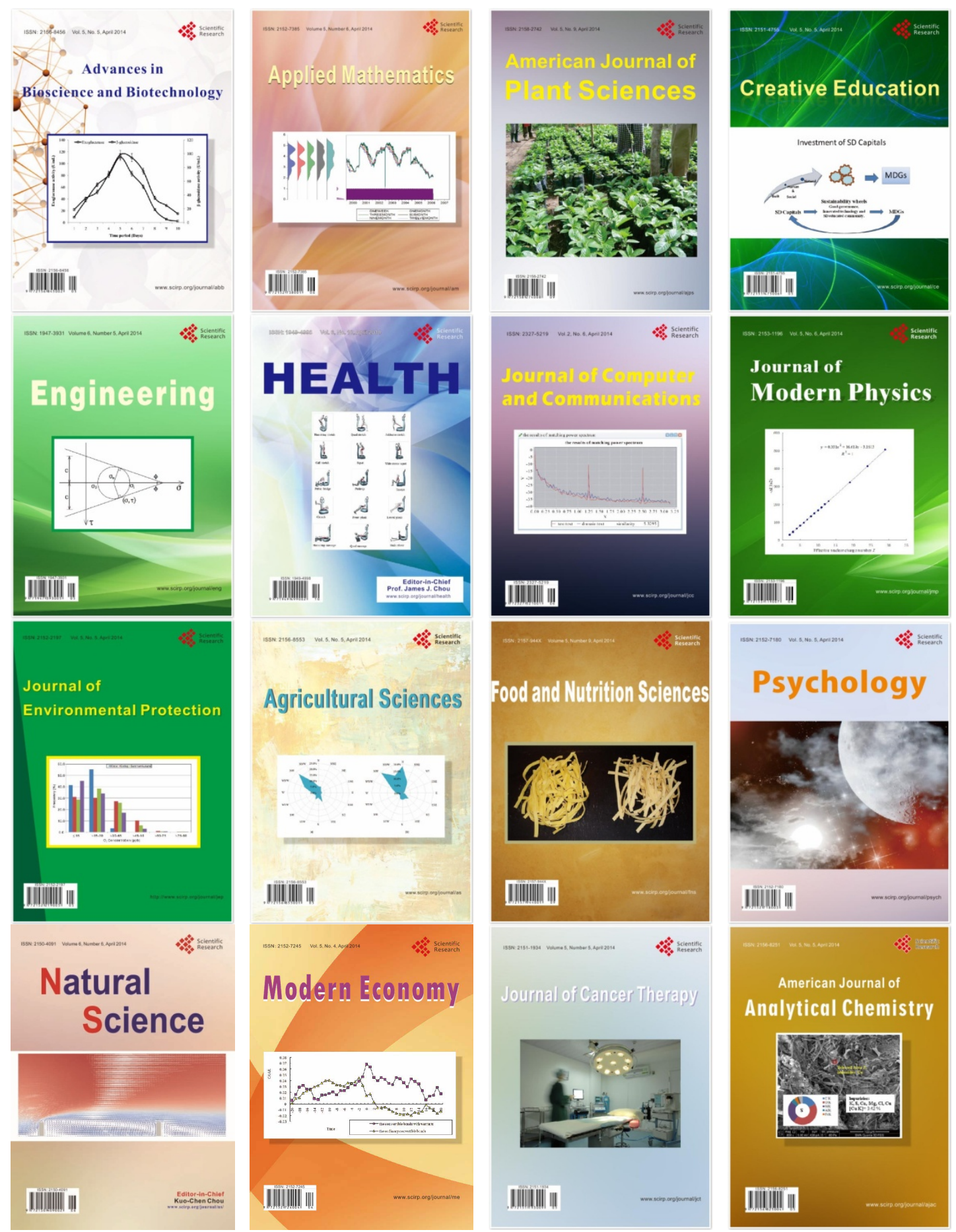